\title{
The Solving Method of Sports Teachers' Work for Data Processing Problem
}

\author{
Bo Zhou ${ }^{1,2 *}$ \\ ${ }^{I}$ Physical Culture College, Hunan Normal University, Changsha 410012, Hunan, China; ${ }^{2}$ Key Laboratory of Physical \\ Fitness and Exercise Rehabilitation of Hunan Province, Changsha 410012, Hunan, China
}

\begin{abstract}
In this paper, the application of computer software and computer network, can solve the bottleneck problem in the process of sports data processing methods, to improve the present stage large physical test and college sports in the efficiency of large amount of data entry, to solve the current implementation of "student physical health standard" and all kinds of large sports data required for testing equipment, shortage of funds and the contradiction between students surge. Male body with university course and information processing network system not only can save a lot of manpower and material resources, and can effectively solve the problem of the bottleneck of data processing, improve the efficiency of PE teachers' work. Using object-oriented technology development is suitable for the control in the field of sports is the key to solve the sports information system suitability. Based on input data of the common sports information system, design the physical data processing control, in the sports teaching management system and the sports qualified standard management system obtain the good use effect.
\end{abstract}

Keywords: College sports, computer software, data processing, physical education teachers, the network system.

\section{INTRODUCTION}

In China, the University physical education is the only door from required of them. With the advent of the era of digital and network, all the activities of human beings by data records, archiving, transmission through the network. And these used to archive data will exercise according to certain rules, data will be according to certain direction, thus formed the data flow. In public physical education in university is also a data stream, the data flow will flow through every link of the public physical education university, the university public physical education play a record, analysis, evaluation, guidance, and so on.

Information system in the field of sports development, according to the specific way of working and one aspect of the specific work for software design is more, so the developed software application range is very narrow, lack of promotion value; Adaptability is bad, once the work has changed, the software is no longer applicable, must be software development, causes the waste of resources. As an information system in the field of sports, often involve a large amount of original data input, conversion and evaluation; Although different sports information systems or different objects within the same system of the need of sports and evaluation standard is different, but in aspects of data structure, internal processing mechanism still has a great deal of commonality, therefore, can through the establishment of the abstract and encapsulate data processing control [1-6]. Object-oriented design emphasizes the model of the problem space, through the abstraction of the same object, the object encapsulates the system internal variable part and relatively stable part of the separation, through the way of inheritance provider code reuse mechanism, can promote the efficiency of software development, improve the quality and maintainability of the system. So using object-oriented technology is suitable for sports development in the field of control is the key to solve the sports information system inadaptable.

\section{PHYSICAL EDUCATION TEACHERS' WORK AND DATA PROCESSING}

Along with the advance of modern science and technology, computer and computer network into people's life, the university public physical education and the students physical health standard test data processing method are also great changes have taken place, like a good statistical software SAS, SPSS, palmpilot type and PDA has fully replace the traditional manual calculation mode. This not only greatly increase the speed of data processing, reduce the human error, more important is to improve the degree of the results of the analysis of "fresh", make the whole sports data statistical process has more practical value.

However, in terms of China's current economic development level, the public physical education in the university and the student physical health standard test electromechanical integration equipment has been widely used in data collection is not realistic; more data collection activities depend on the traditional equipment. And when using traditional equipment for data acquisition, usually to use paper data table to record the data, in order to get a complete test results. For the convenience of calculation, the data must be into the computer. To make the data from the form of paper 
Table 1. The scores of physical inspection items in colleges and universities.

\begin{tabular}{|c|c|c|c|c|c|c|c|}
\hline \multirow{2}{*}{ Projects } & \multirow{2}{*}{ Gender } & \multicolumn{2}{|c|}{ Excellence } & \multicolumn{2}{|c|}{ Well } & \multicolumn{2}{|c|}{ Medium } \\
\hline & & Performance & Score & Performance & Score & Performance & Score \\
\hline \multirow{2}{*}{$50 \mathrm{~m}$} & male & $6.8 \mathrm{~s}$ & 30 & $6.9-7.3 \mathrm{~s}$ & 26 & $7.4-7.7 \mathrm{~s}$ & 23 \\
\hline & female & $8.3 \mathrm{~s}$ & 30 & $8.4-9.1 \mathrm{~s}$ & 26 & $9.2-9.6 \mathrm{~s}$ & 23 \\
\hline $1000 \mathrm{~m}$ & male & $3.39 \mathrm{~min}$ & 20 & $3.4-4.0 \mathrm{~min}$ & 16 & $4.01-4.18 \mathrm{~min}$ & 15 \\
\hline $800 \mathrm{~m}$ & female & $3.37 \mathrm{~min}$ & 20 & $3.38-4.0 \mathrm{~min}$ & 16 & $4.01-4.19 \mathrm{~min}$ & 15 \\
\hline \multirow{4}{*}{ swimming } & male & $150 \mathrm{~m}$ & 20 & $80-100 \mathrm{~m}$ & 16 & $40 \mathrm{~m}$ & 15 \\
\hline & & $200 \mathrm{~m}$ & 20 & $100-150 \mathrm{~m}$ & 16 & $60 \mathrm{~m}$ & 15 \\
\hline & female & $80 \mathrm{~m}$ & 20 & $60-40 \mathrm{~m}$ & 16 & $20 \mathrm{~m}$ & 15 \\
\hline & & $100 \mathrm{~m}$ & 20 & $60-80 \mathrm{~m}$ & 16 & $40 \mathrm{~m}$ & 15 \\
\hline learning attitude & & & $9-10$ & & $8-8.9$ & & $7-7.9$ \\
\hline basic knowledge of sport & & & $9-10$ & & $8-8.9$ & & $7-7.9$ \\
\hline physical healthy standard & & \multicolumn{2}{|c|}{$\begin{array}{c}86-100 \text { score } \\
10\end{array}$} & \multicolumn{2}{|c|}{$\begin{array}{c}76-85 \text { score } \\
8\end{array}$} & \multicolumn{2}{|c|}{$\begin{array}{c}65-75 \text { score } \\
6\end{array}$} \\
\hline
\end{tabular}

that existed in a computer data entry process. University public physical education and the student physical health standard test data are often large quantities of data, such as a university public physical education in the first and second grade as the compulsory, grade three and grade four for elective, appraisal for the structural test.

Assessment type is divided into examination of the term, academic year examination, examination, to take grade ratings, exams and converted into specific percentile. Type and examination, examination subjects in Table $\mathbf{1}$, which is divided into score, such as learning attitude accounts for 10 points, basic knowledge of sports accounts for 10 points, accounts for 10 points of student physique healthy standard and the non-score $(50 \mathrm{~m}$ and $800 \mathrm{~m}$ for minutes, swimming in terms of $\mathrm{m}$, according to the different period (such as the SARS epidemic period) examination and subject to change, the teacher wants to Table $\mathbf{1}$ will not scale data are converted to score first, which makes the teacher's work a lot with in minutes on m value is converted to a percentile; Additionally according to the latest "student physical health standard" formulated by the national ministry of education, every student to test at least seven projects, each student at least seven statistics; All 10000 college students, to conduct a "student physical health standard" test, 70000 data should be collected at least, not including the student's basic information, such as name, student number, gender, etc. Assumes that the operator can accord the data/20 min speed (this is the very high input speed) entry, 3 min 50070000 data required to input. As an organism, its attention to keep the highly centralized maximum time of 45 minutes, then need to rest and adjustment. Per person per day can be entry time of $270 \mathrm{~min}$ and 70000 data need 13 days to complete work of the input, and data entry and all data after complete programming minute's $m$ value to the percentile of transformation. But the 70000 data analysis of mathematical statistics process takes less than a few minutes. By extension, in the national large physical detection, comparable amount data collection is by no means such a school, it is not surprising that millions or even thousands of data. The 13 days and a few minutes of gap, the greater the amount of data, its disparity also will be greater. Thus, data input speed and data conversion has become the biggest barrier to seriously affect the physical data stream processing, is the bottleneck of restricting the sports information.

\section{THE LOSS OF DATA BOTTLENECK AND DATA ENTRY PROCESS}

A teacher at the end of the semester in a lot of time and energy is from on the convert data to score. At the end of the semester, in the teaching process, teachers should be a summary of this semester teaching work, but that is not the case, so that teachers don't have the time and effort to timely summary this semester teaching and the problems existing in the practice; From the perspective of process control, collection of university physical education and the students physical health standard test data has guiding significance for the process of physical education is to provide information. For a team's training, coaches spent a lot of energy to collect data, and its purpose is to want to analyze these data, learn about training process. Such as how the effects of training, there are the problems in training, each player's progress in size, etc., make the results of data analysis can be helpful to the training or guidance. Similarly national youth and physical investigation in order to master Chinese teenagers' physique condition, analysis of the living environment and economic development, natural environment on the influence of the adolescent growth and development, find out the effective way to improve the adolescent growth and development environment, to a more healthy growth [7].

However, due to the speed of data entry and data transformation, a large amount of data delay in the entry and 
transformation stage, for a team, can bring only training efficiency is not high, defeat in the race, the loss is nothing but hundreds of thousands, millions of money. If it is delayed due to the problem of data entry guide teenagers for a generation of growth and development, the loss is immeasurable.

After the original data collection, data entry, people is through the eyes of recognition to the Numbers on the paper form through the eye, brain, hand, keyboard, digital into the process, in the digital signal into computer input data at the same time also subject to the conditions of the time and place. In a nutshell, is as an organism, think of themselves as a signal conversion tool, will be able to identify our human signal, signal into computer can identify and storage process.

Look at the process of data transformation and data by means of artificial control form converted to score, teachers' workload and error-prone, to convert the data is read in paper form data, contrast the physique health standard ", through the processing in the brain, and then converts first-hand data to score, command their own hands to fill in the figure, the work is tedious and meaningless.

Human as an organism, the comprehensive analysis of information ability is very excellent, but in the mechanical processing information ability, it is far less memory capacity of computer and computer network. Will is a must to increase the speed of data entry, "low energy" "people" liberated from the data entry and transformation process, with high data transmission ability of the computer network and computer to replace. Computer network can make a lot of data entry assigned to all the teachers from different locations, different time for data transmission; it can be a lot to save time. The transformation of the data available establishes the university male body information processing network system, and a computer program solves this problem.

University of male body class information processing network system will have the following functions: the network transmission function; can be set up according to the different grade, the different period is not the same examination subjects; with converts min, s, m score function, at the same time can change according to time and the score table and change; with long-term storage function; Cost is not higher than the cost of using paper form; With functions of data statistics.

\section{THE USE CONDITIONS AND METHODS OF COMPUTER NETWORK COURSEWARE}

The basic principle includes general principles, controllability principle, and the scientific principle. Media selection and combination of optimization principle is friendly interface, easy operation principle. Sports technology uses the superiority of multimedia teaching courseware, which is beneficial to difference teaching, according to their aptitude. It is conducive to good teaching experience, the results of promotion. To cultivate the students' intelligence factors and non-intelligence factors. Is beneficial to teachers strength and energy saving, ensure that teachers have a good mental state to motivation, creatively. To cultivate students' sensitive consciousness of modern science and technology, we promote the formation of the creative thinking [8-13].
The most simple terms, in one or several surrounding the track can undertake multimedia teaching classroom, the classroom at least a 7-day containing more than 486 computer drive, sound card, and have an external speaker, large screen color TV one or part of the projector, so that we can satisfy teachers' presentation of multimedia courseware. In order to more fully use of multimedia courseware teaching, in addition to the above mentioned conditions, the classroom must also establish a local area network system, under the management of the server, there are 30 or so computer, which can satisfy the 20 to 30 people at the same time use the computer self-study or tutorial. the best condition, in addition to access to multimedia courseware in class, students in their spare can also make use of multimedia courseware for preview or review consolidate, which requires students' dormitory equipped with computer or open to students at any time of the multimedia classroom. With the development of science and technology level and the improvement of people's living standard, the computer prices are falling sharply, which has for the sports multimedia teaching provides a feasible technology courses teaching conditions.

Have the basic hardware facilities, software to keep up with in a timely manner. It mainly contains two meanings: one is the basic requirements for teachers and students, teachers and students should have basic computer knowledge and use of basic skills, such as modern media and the camera can open and use computer, for example, and broadcasting multimedia courseware and how to use camera students technical movement, etc.; it is to have a good multimedia courseware, it is a "source" of the problem, if no good multimedia courseware, it is a good hardware can play a role.

In order to achieve good teaching effect, we are usually in the teaching process, students are required to learn to preview before class, class, after class to consolidate. Use of multimedia courseware for teaching, but also should give priority to with the three teaching links in teaching (Fig. 1).

\section{THE REALIZATION AND ANALYSIS OF THE NETWORK SYSTEM ENVISAGED}

\subsection{The Realization of the Idea}

Public body set up under the university course and information processing network system model (see Fig. 2). Using ASP writes a computer program to establish the university public class information processing network system. ASP (Active Server Pages) is written to a powerful network application and realization of Server data processing and the function of the upload and download, at the same time, ASP technology has the following characteristics.

(1) Using VBScript, JavaScript and other simple script, combined with the HTML code, can quickly complete the development of dynamic web pages.

(2) Do not need to compile, easy to write, can run on the server side.

(3) Use plain text editor, you can edit the design.

(4) Has nothing to do with the browser. Client with HTML browser, can the design by ASP website content. Used in ASP script, such as VBScript, JavaScript, are available on 


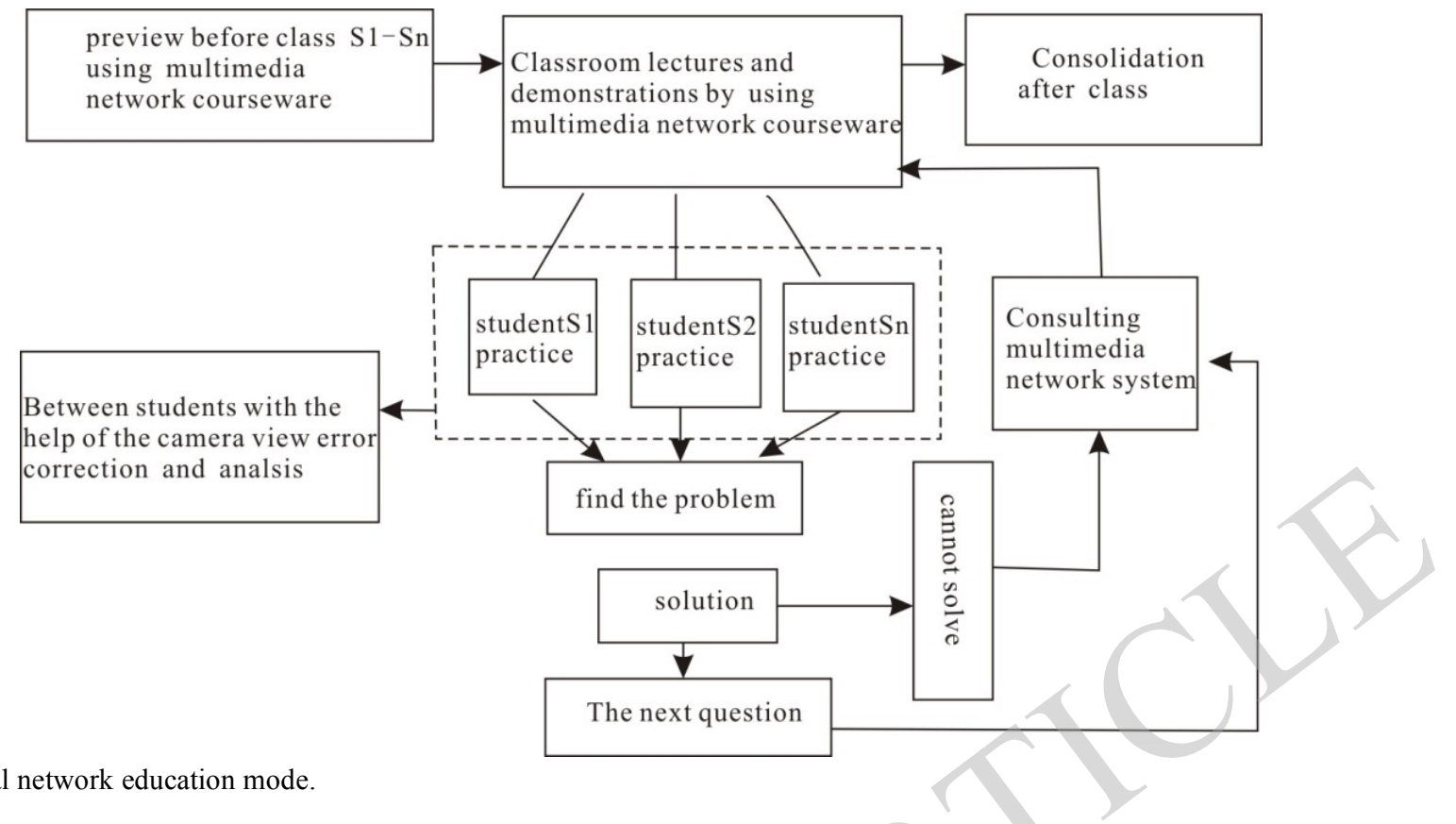

Fig. (1). Physical network education mode.

the Web server, client browser cannot have the ability to run the script.

(5) ASP compatible with any ActiveX Scripting language.

(6) ASP source code won't spread to the client browser, thus can avoid source code is written by people of plagiarism, improved the security of the program.

(7) Can use a server-side script to generate the client script. ity.

(8) ActiveX Server Components have unlimited scalabil-

The multimedia network teaching platform using the latest $\mathrm{B} / \mathrm{S}$ (browser/server) structure, its structure is shown in (Fig. 2). The characteristics of the structure is as follows: the client application environment for the standardized general Web browser, all the applications are stored on a Web server, when need can be directly downloaded; More easy to manage and maintain, due to the client without special software, when to upgrade network applications, only need to update the server software can; This structure has good expansibility, openness, $\mathrm{B} / \mathrm{S}$ structure with a standard TCP/IP communication protocol, the school can according to their own development needs to expand the system at any time.

Working principle is teachers and students through the browser to access the multimedia network teaching platform, students use personal computer equipment connected to the server via a browser, carries on the related sports in teaching contents and learning resource information query, the timely communication between teachers and students, such as personal data uploading operation. Multimedia network teaching platform of management and college sports teachers can update content stored in the server through the browser and the maintenance, and will the latest sports teaching resource information uploaded to the server side, at the same time can be achieved with the student online question, and can make sports guidance to students. Server consists of a Web server and database server. With a Web server to store all kinds of application modules to the system, complete the application functions of customer, it receives the user's request, the client and translated into a database request after interaction with the database server, and the interaction results in the form of a Web page to download to the browser, users can request the results observed. Database server storage required by the system database and its management software, it according to the request from the Web server for database operation, and the results to the Web server.

The information processing system adopts three layers $\mathrm{B} / \mathrm{S}$ architecture, with Microsoft. Net as a platform, for each local project server, they are responsible for the local data processing work. By points, calculation of the input, and then get the result and the corresponding reports. In addition, you also need to pass the result to the data center server, to generate the statistics table. The data center server saved up from each subproject upload of data, and then on this basis, update web site, visitors to query for society. Each component systems are typical three layers B/S architecture. (Fig. 3) sports result processing system for colleges and universities.

\subsection{Web Service}

Game of integrated data released in the center of the Shanghai university server, all data and reports from various colleges and universities to upload to the center of Shanghai university server, the network transmission system to choose the Webserver ice, it is Microsoft now praises a new network transmission technology, by adding the corresponding Web reference, make local process used in remote server defined method as simple as using local methods. And Web Service in the lower layer using the Soap protocol to transmit XML format information, has the very good stability and compatibility. Web Service architecture defines three kinds of roles and role between the three kinds of operation, as shown in Fig. (4). 


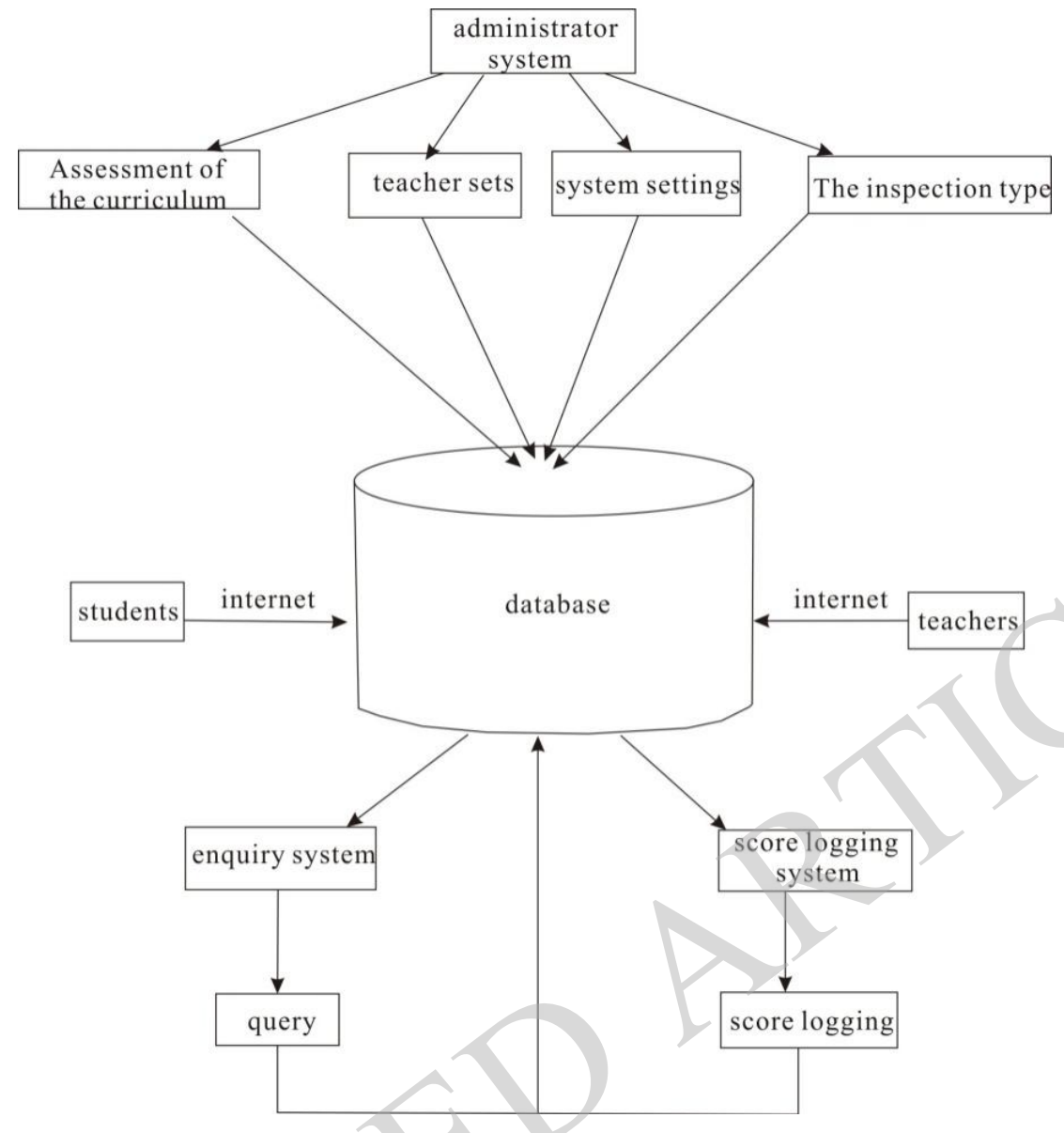

Fig. (2). System model of information processing network of physical education.

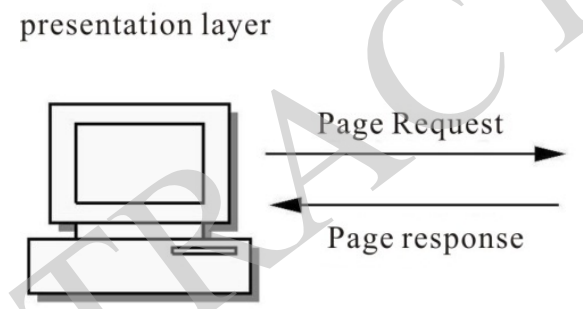

browser logical layer

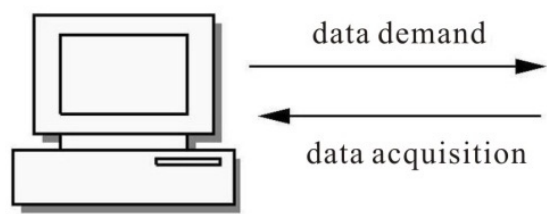

DB server

Fig. (3). Schematic diagram of physical teaching process system.

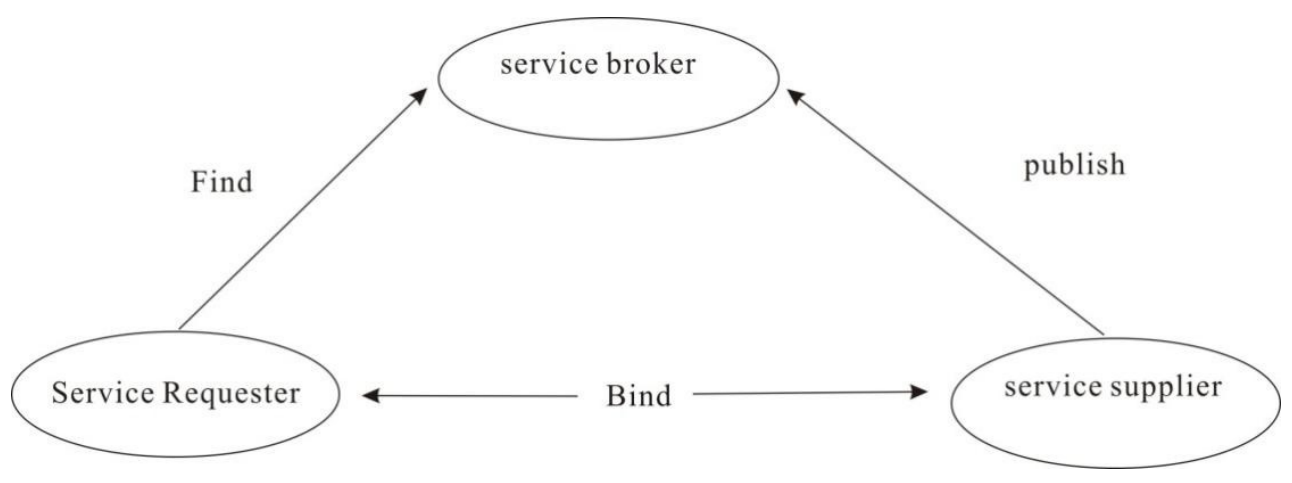

Fig. (4). System structure diagram of web service. 
Service requesters and service providers in the system to know each other, don't need through a service agency to provide registration, classification and search service, so you can put the Web Service system structure is simplified into only service requesters and service providers, provided by the back-end data center services, distribution in various colleges and universities of ASP. NET application invokes the service.

\subsection{Result Analysis}

A school data manually input need 13 days to complete, so national large physical testing data will need how many days? In 1985, constitution detection took 2 years to get out the statistical results after the fastest results in more than six months. Imagine two years before he came to the conclusion, the conclusion of timeliness, its realistic guiding significance to also sell at a discount greatly.

If university public class information processing network system is used to record the acquisition of data, and by more than ten minutes or a few minutes to solve the half a year or two years to solve the problem, its efficiency is proved; Besides this way of data input and transformation is pure electronic means, avoid the person's participation, its transmission rate in at least up to $512 \mathrm{Kbit} / \mathrm{s}$, namely to send 512 Kbytes per second, for it is not human. If further data statistics, the statistical results on most days can this half a year enough to their days fully proved that the advanced nature of the new technology.

\section{CONCLUSION}

It is not hard to see from the analysis of the section on, uses the modern network technology, the process is not only an entry, actually contains the data entry and statistical calculation and analysis, signal conversion process. It is too manually, data conversion and spread to the general statistical process testing personnel in the process of using computer network and computer. This not only can greatly improve the data entry speed, greatly reduce the working pressure of entry personnel, the more important is adopted the advanced network system, can improve the efficiency of the data and statistical results and improve the results to the real work ability and guide the direction of the accuracy of the cost in the male body class examination and physical testing or training and teaching in the test cost value. At the same time, due to the use of the male body class information processing network system, make the whole process reduced the usage of paper, environmentally friendly. So as the following suggestions is shown.

(1) Given the powerful computers and computer network data entry, data transformation and data statistical power, suggested that widely used in the process of sports data collection information processing network system, the course of college and university public body for the speeding up of the data input, transformation, and statistics, solve the bottleneck of data flow.

(2) From the perspective of environmental protection, it is recommended that the adoption of new technology as soon as possible, realize the sports work paperless, make the new technology to make due contributions to sustainable development.

\section{CONFLICT OF INTEREST}

The author confirms that this article content has no conflict of interest.

\section{ACKNOWLEDGEMENTS}

Declared none.

\section{REFERENCES}

[1] C.V.C. Bouten, K. Koekkoek, M. Verduin, R. Kodde and J.D. Janssen, "A triaxial accelerometer and portable data processing unit for the assessment of daily physical activity", IEEE Transactions on Biomedical Engineering, vol. 44, no. 3, pp. 136-147, 1997.

[2] M. Sperber, "College sports inc.: the athletic department vs. the university", Phi Delta Kappan, vol. 72, no. 2, 1990.

[3] M. Papastergiou, "Exploring the potential of computer and video games for health and physical education: A literature review", Computers \& Education, vol. 53, no. 3, pp. 603-622, 2009.

[4] W. Kroeze, A. Werkman and J. Brug, "A systematic review of randomized trials on the effectiveness of computer-tailored education on physical activity and dietary behaviors", Annals of Behavioral Medicine, vol. 31, no. 3, pp. 205-223, 2006.

[5] R. McKethan, B. Everhart and R. Sanders, "The effects of multimedia software instruction and lecture-based instruction on learning and teaching cues of manipulative skills on preserve physical education teachers", Physical Educator, vol. 58, no. 1, p. 2, 2001.

[6] M. Standage, J.L. Duda and N. Ntoumanis, "A model of contextual motivation in physical education: Using constructs from selfdetermination and achievement goal theories to predict physical activity intentions", Journal of Educational Psychology, vol. 95, no. 1, p. 97, 2003.

[7] D.A. Phillips, C. Carlisle and J. Steffen The Physical Education Teacher Assessment Instrument: Computerized Version [Computer Software]. Greeley, CO: University of Northern Colorado, 1988.

[8] B. Mohnsen, "Using instructional software to meet national physical education standards" Journal of Physical Education, Recreation \& Dance, vol. 72, no. 3, pp. 19-22, 2001.

[9] M. Stanescu, M. Stoicescu and C. Ciolca, Computer use in physical education and sports teaching, $7^{\text {th }}$ Scientific International Conference Elearning and Software for Education, Bucharest, 28-29, 2011.

[10] X. Zhang and J. Chen, "The developing and implementing of multimedia physical education CAI software", China Sport Science and Technology, vol. 6, p. 020, 2002.

[11] P. Hockicko, "Useful computer software for physical analysis of processes", Proceedings of the 2009 Information and Communication Technology in Education, Annual Conference, $15^{\text {th }}-17^{\text {th }}$ September 2009.

[12] A. Vuillemin, F. Guillemin, G. Denis, J. Huot and C. Jeandel, “A computer-assisted assessment of lifetime physical activity: reliability and validity of the QUANTAP software" Revue d Epidemiologie et de Santé Publique, vol. 48, no. 2, pp. 157-167, 2000.

[13] C. Wilkinson, T.R. Pennington, and G. Padfield, "Student perceptions of using skills software in physical education", Journal of Physical Education, Recreation \& Dance, vol. 71, no. 6, pp. 37-40, 2000. 